\title{
Rayleigh-Bénard convection, thirty years of experimental, theoretical, and modeling work
}

Paul Manneville

Laboratoire d'Hydrodynamique, École polytechnique

F-91128 Palaiseau cedex, France

e-mail: paul.manneville@ladhyx.polytechnique.fr

\begin{abstract}
A brief review of Rayleigh-Bénard studies performed all along the twentieth century is presented, with an emphasis on the transition to turbulence and the appropriate theoretical framework, relying on the strength of confinement effects and the distance to threshold, either dynamical systems for temporal chaos in the strongly confined case, or models of space-time chaos when confinement effects are weak.
\end{abstract}

\section{Introduction}

The idea of convection is quite old (Hadley, Lomonossov, Rumford,...) but the first quantitative experiments have been performed by Henri Bénard around year 1900 [1]. Figure 1 is a low-resolution reproduction of one of his original photographs. In fact Bénard studied the stability of a thin fluid layer open to air and submitted to a vertical temperature gradient. He accurately determined properties such as the space periodicity of the hexagonal pattern, its variation, the profile of the interface. Later, in 1916, Lord Rayleigh [2] proposed his theory of a feedback coupling resting on buoyancy: a fluid particle hotter than its

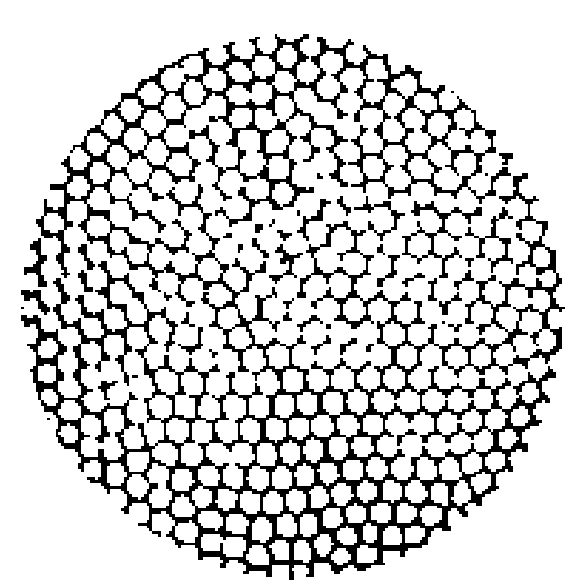

Fig. 1. One of Bénard's celebrated original photographs of the top view of convection patterns in a thin layer of spermaceti heated from below.

\section{Dynamics of Spatio-Temporal Cellular Structures Henri Bénard Centenary Review}

I. Mutabazi, E. Guyon, J.E. Wesfreid, eds. Springer Tracts in Modern Physics

(Springer, 200x) 

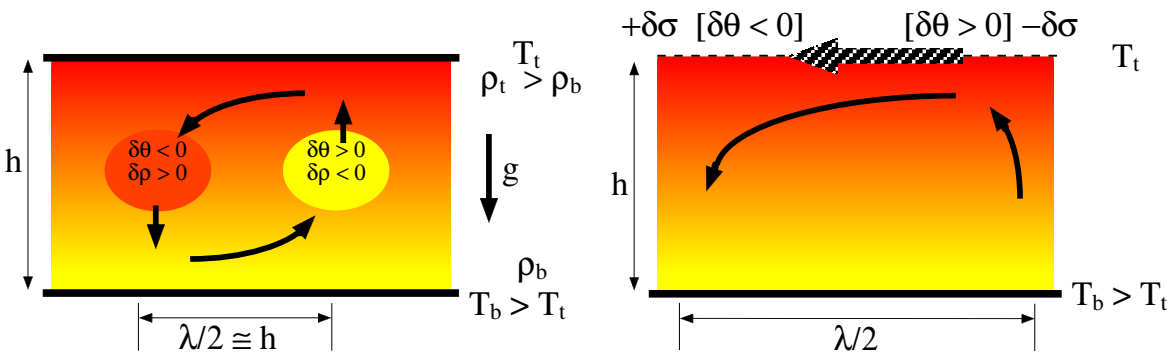

Fig. 2. Mechanisms for Bénard convection. Left: motion of fluid is self-sustained as soon as gravitational energy release can overcomes dissipation losses (Rayleigh's idea). Right: the surface-tension mechanism (Marangoni effect) works even in zero-gravity environment provided that hot fluid comes from inside.

environment encounters ever colder fluid as it rises, which leads to the instability, as sketched in Fig. 2 (left). He developed a complete linear stability analysis assuming stress-free conditions for the velocity and good heat-conducting plates. This mechanism was accepted as the explanation of Bénard's results until the role of the thermal Marangoni effect was pointed out, in particular by Pearson [3]: a temperature fluctuation at the surface induces tangential stresses that can be amplified by hot fluid coming from the inside, as suggested in Fig. 2 (right). Surface tension usually increases as the temperature decreases while surface elements with the larger surface tension want to shrink, so that the mechanism indeed works when the temperature gradient is directed towards the interior of the layer. Some of the confusion may be explained by the fact that hexagons are usually expected when the top-bottom symmetry is broken, i.e. when boundary conditions at the top plate are different from those at the bottom plate, which was the case of Bénard's experiments with a free upper surface. In that case, the two mechanisms are in competition but a bump is implied at the place where the fluid rises when the buoyancy is involved, whereas the effect of surface-tension is to create a dip. Buoyancy effect in the bulk will dominates in thick layers and surface-tension in thin ones so that there exists a compensation thickness at which both deformations balance each other [4]. This being recognized, in the following I will restrict myself to the consideration of convection between solid plates according to Rayleigh's mechanism, hereafter called $R B$ convection, as opposed to BM convection, where ' $\mathrm{R}$ ' stands for Rayleigh, ' $\mathrm{B}$ ' for Bénard, and 'M' for Marangoni.

I will briefly recall the results of linear stability analysis in $\S 2$, and then some early nonlinear findings about secondary instabilities and the transition to turbulence in $\S 3$. After these preliminaries I will mainly turn to modeling issues raised by the theoretical understanding of the results beyond threshold. As to weakly turbulent states developing moderately far from the threshold, I will insist on the role of confinement effects in controlling the nature of scenarios either toward chaos as envisioned in dynamical systems theory ( $\S 4$ ) or towards spacetime chaos $(\S 5,6,7)$. Before closing the presentation I will also say few words 
about the account of strongly turbulent sates when the applied temperature gradient becomes arbitrarily large in $\S 8$.

The present review is of course sketchy and biased by my personal interests. For further information and complementary views, the reader is invited to consult the works mentioned in the bibliography. An early self-contained presentation is in the first chapters of Chandrasekhar's book [5]. A more recent general reference is by Koschmieder [6]. Consult also the reviews by Busse [7] about nonlinear convection, by Newell, Passot and Lega [8] and Cross and Hohenberg [9] for the theoretical approach of space-time chaos with emphasis on the envelope formalism and general aspects of space-time chaos respectively. The most recent developments about the transition to turbulence in convection can be found in the review by Bodenschatz, Pesch and Ahlers [10], while the article by Siggia $[11]$ is devoted to fully developed turbulent convection.

\section{$2 \quad$ RB convection at threshold}

Rayleigh's theory for the instability threshold ${ }^{1}$ was developed within the socalled Boussinesq approximation, i.e. the Navier-Stokes equations for an incompressible flow completed by the energy equation generalizing the Fourier diffusion equation to a fluid medium. The simplifying assumption is that the temperature only enters the state equation to account for thermal expansion, while all other fluid parameters are kept constant. Within this approximation the governing equations read:

$$
\begin{aligned}
& \nabla_{\mathrm{h}} \cdot \mathbf{v}_{\mathrm{h}}+\partial_{z} v_{z}=0 \\
& P^{-1}\left[\left(\partial_{t}+\mathbf{v} \cdot \boldsymbol{\nabla}\right) \mathbf{v}_{\mathrm{h}}+\nabla_{\mathrm{h}} p\right]=\nabla^{2} \mathbf{v}_{\mathrm{h}} \\
& P^{-1}\left[\left(\partial_{t}+\mathbf{v} \cdot \boldsymbol{\nabla}\right) v_{z}+\partial_{z} p\right]=\left(\nabla^{2} v_{z}+\theta\right) \\
& \left(\partial_{t}+\mathbf{v} \cdot \boldsymbol{\nabla}\right) \theta=\nabla^{2} \theta+R v_{z}
\end{aligned}
$$

These equations are written here in dimensionless form for the perturbation around the basic state. Coordinate $z$ is along the vertical and subscript ' $\mathrm{h}$ ' indicates the horizontal directions. The temperature fluctuation $\theta$ is defined as the departure from the linear temperature profile given by the Fourier law in the fluid at rest: $T_{0}(z)=T_{\mathrm{b}}-z \Delta T / h$ ( $h$ is the height of the cell and $\Delta T=T_{\mathrm{b}}-T_{\mathrm{t}}$ is the temperature difference between the bottom and top plates). The natural control parameter is the Rayleigh number defined as:

$$
R=\frac{\alpha g \Delta T h^{3}}{\kappa \nu}
$$

where $\alpha$ is the expansion coefficient and $g$ the acceleration of gravity. The thermal diffusivity $\kappa$ and the kinematic viscosity $\nu$ parameterize the stabilizing dissipative processes. The last parameter that shows up in these dimensionless equations is the Prandtl number $P=\nu / \kappa$ that controls the nature, either mostly thermal or

\footnotetext{
${ }^{1}$ The material in this section is discussed at length in Chandrasekhar's book [5].
} 
rather hydrodynamic, of the physical processes at stake, as they depend on the relaxation rates of temperature and vorticity fluctuations. Equation (1) accounts for the continuity of the flow and (4) for heat conduction in the fluid. Differential buoyancy shows up in (3) through the term in $\theta$ and, at this stage the horizontal component of the Navier-Stokes equation (2) only plays a passive role (closing of flow lines). Boundary conditions must be added to these equations. The cleanest situation is for good conducting rigid (i.e. non slip) plates, which yields:

$$
\theta\left(z=z_{\mathrm{p}}\right)=0 \quad \text { and } \quad \mathbf{v}\left(z=z_{\mathrm{p}}\right)=\mathbf{0}
$$

where $z_{\mathrm{p}}$ denotes the plate's position. As already mentioned, Rayleigh assumed stress-free boundary conditions at top and bottom, hence:

$$
\theta\left(z=z_{\mathrm{p}}\right)=0 \quad \text { and } \quad v_{z}\left(z=z_{\mathrm{p}}\right)=0, \quad \nabla_{\mathrm{h}} \mathbf{v}_{\mathrm{h}}\left(z=z_{\mathrm{p}}\right)=\mathbf{0}
$$

He solved the problem for Fourier normal modes in the form $\propto \exp \left(i \mathbf{k}_{\mathrm{h}} \cdot \mathbf{x}_{\mathrm{h}}\right)$ where all the functional dependence in the vertical coordinate are absorbed in the proportionality sign. A straightforward calculation from the Boussinesq equations (1-4) and boundary condition (6) yields the marginal stability condition:

$$
R_{\mathrm{m}}(k)=\frac{\left(k^{2}+\pi^{2}\right)^{3}}{k^{2}},
$$

where $k$ is the length of the horizontal wave-vector $\mathbf{k}_{\mathrm{h}}$. This curve is displayed in Fig. 3, together with that corresponding to the more realistic no-slip velocity boundary conditions (5) obtained by Pellew \& Southwell (1940). The minimum of each curve defines the corresponding threshold above which convection sets in, $R_{\mathrm{c}}=27 \pi^{4} / 4 \simeq 657.5$ and $R_{\mathrm{c}} \simeq 1708$ for stress-free and no-slip conditions

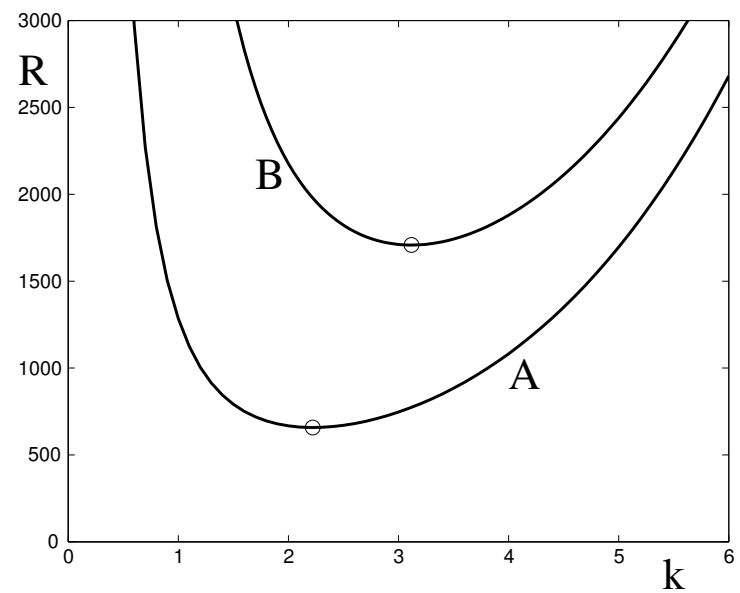

Fig. 3. Marginal stability curves for stress-free (A) and no-slip (B) velocity boundary conditions and isothermal plates (infinitely large conductivity). 
respectively. The expected diameter of the convection cells is half the critical wave-length $\lambda_{\mathrm{c}}=2 \pi / k_{\mathrm{c}}$. In the no-slip case $k_{\mathrm{c}} \simeq 3.12$, which make the predicted diameter very close to the height of the cell $(\pi / 3.12 \simeq 1.007)$. In the stress-free case one obtains $k_{\mathrm{c}}=\pi / \sqrt{2}$ which gives a somewhat larger diameter $(\sqrt{2} \simeq 1.4)$. This prediction could have partly been responsible for the confusion alluded to above because it was close to Bénard's observations (but for a Marangonibased mechanism) though, strictly speaking, one should have compared with the prediction for mixed boundary conditions (bottom: no-slip, top: free) that yields $R_{\mathrm{c}} \simeq 1101$ and $k_{\mathrm{c}} \simeq 2.68$. [Of course other cases can also be studied, especially by relaxing the assumption about the thermal conductivity of the plates.]

\section{$3 \quad$ RB convection beyond threshold}

As the Rayleigh number exceeds the threshold value, part of the heat is transported by convection which decreases the potential for instability, while thermal diffusion and viscous friction increases due to the horizontal gradients implied by the modulation. This explains that the amplitude of the motions saturates beyond threshold. For rolls, the bifurcation turns out to be supercritical, i.e. behaves continuously in the vicinity of the threshold and the system builds up a well defined steady pattern. Early studies have been devoted to the problem of which kind of periodic pattern was selected by nonlinearities, rolls made of a single pair of wavevectors $\pm k \hat{\mathbf{x}}$ where $\hat{\mathbf{x}}$ is a unit vector in the horizontal plane, squares made of two pairs of wavevectors at right angles, hexagons with three pairs at $120^{\circ}$ (Fig. 4). The theory was again first developed for stress-free

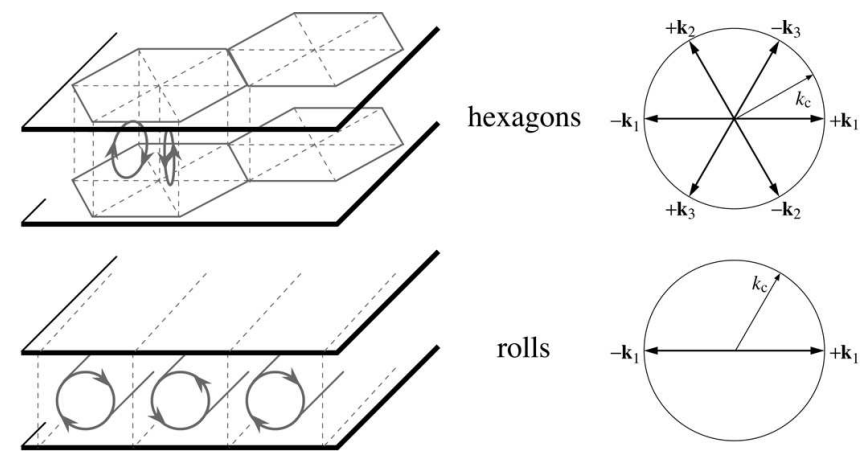

Fig. 4. Hexagons vs. rolls. Hexagons result from the superposition of three pairs of modes at $120^{\circ}$. Those appearing in thin convecting layer are produced by the Marangoni effect and have fluid sinking in the center of the cells. In thicker layers, the Rayleigh mechanism produce hexagons with fluid sinking at the cell edges. Both bifurcate subcritically. Rolls are obtained from a pair of modes with opposite wavevectors which may point to any direction in the horizontal plane owing to orientational degeneracy in laterally unbounded layers. This degeneracy is then broken by the developing mode. Rolls bifurcate supercritically. 


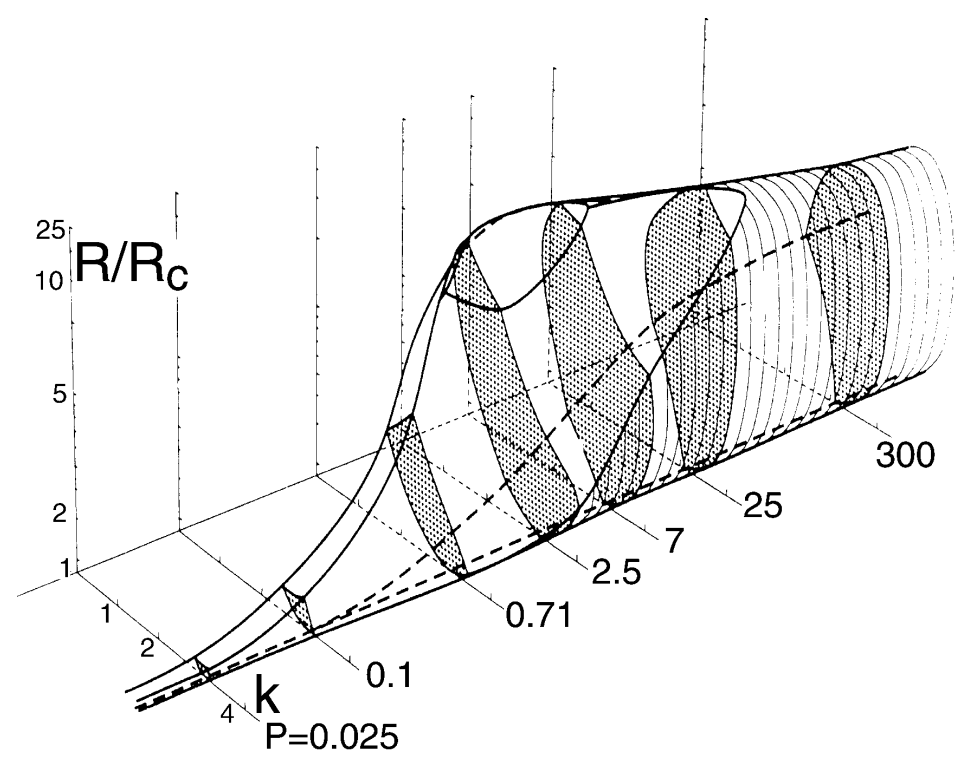

Fig. 5. Busse balloon in perspective in the $(k, P, R)$-space, after Busse [7].

boundary conditions (Malkus \& Veronis, 1958 [12]) and later in the no-slip case (Schlüter, Lortz \& Busse, 1965 [13]), both studies concluding for roll patterns. Subsequent developments concerning the stability of the rolls against various secondary mechanisms mainly belong to Busse and Clever [7]. The result is a surface in parameter space called the Busse balloon separating stable roll patterns from unstable ones. In addition to the Rayleigh number, stability depends on the wavelength of the cells and on the Prandtl number. Some secondary modes are universal, i.e. do not depend on the fact that the pattern is generated by the RB mechanism but on the symmetries of the rolls (invariance through translation $\rightarrow$ Eckhaus instability, and rotation $\rightarrow$ zigzag instability) or on the fact that the intensity of the convection is weak close to the marginal curve (cross-roll instability). Other secondary modes are much more specific to convection, with structures that strongly depend on the value of $P$, e.g. the bimodal instability with secondary rolls localized in thermal boundary layers at right angles with the primary pattern when $P$ is large. Fig. 5 displays a picture of the Busse balloon.

Secondary instabilities are just a step towards more complex behavior as $R$ is increased. Different scenarios have indeed been observed, depending on the value of $P$. Beyond the primary instability leading to the formation of timeindependent two-dimensional ${ }^{2}$ rolls, time dependence was observed to introduce itself first and at relatively low $R$ when $P$ is small, but only after secondary instabilities adding space dependence along the rolls (three-dimensional time independent states) at large $P$ at higher $R$. A compilation of early experimental

\footnotetext{
2 i.e. locally functions of only one horizontal coordinate, say $x$, in addition to $z$.
} 


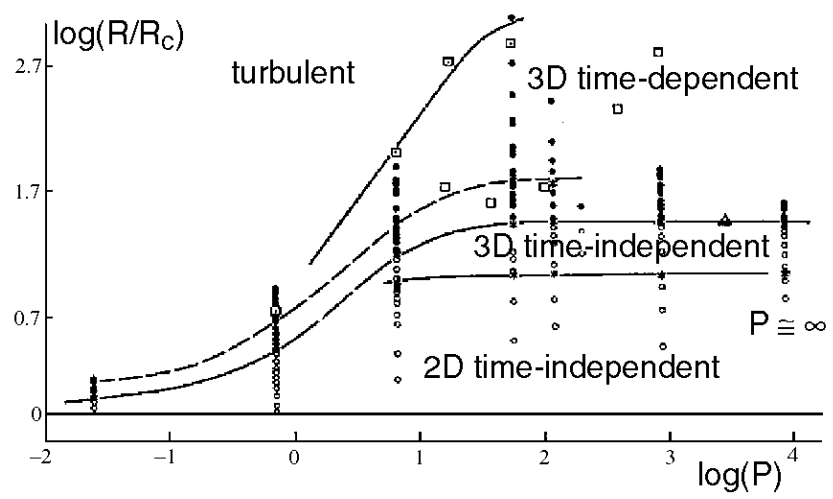

Fig. 6. Transition to turbulence in RB convection: experimental results collected before 1973 by Krishnamurti [14].

results, adapted from Krishnamurti [14], is displayed in Fig. 6.

In all cases the regime called 'turbulent convection' was reached after a finite number of steps. At first sight this fact seemed to support the revision by Ruelle and Takens [15] of the classical Landau theory of transition to turbulence [16]: three or four bifurcations before unpredictable behavior instead of $n$-periodicity resulting from an infinite cascade of Hopf bifurcations. However the situation was not as satisfactory as one would have liked because the thresholds of the observed transitions were not always well defined and a residual, more or less random, component of the time dependence was most often recorded before it was decided to consider the system as turbulent. This was due to the fact that experiments were performed in wide containers, i.e. with many wavelengths, while the transitions were most sensitive to defects in the roll patterns. For example, a state observed at higher $R$ could anticipate transition by nucleating at dislocations. Checking the Ruelle-Takens dynamical systems approach more carefully thus required a better control of the patterns. This has been achieved by playing with confinement effects: only a small number of configuration is available when the number of cells is limited, which makes the Ruelle-Takens approach more relevant a priori, as exemplified in the following section. The case of weakly confined systems will be reviewed next.

\section{Weak turbulence in confined systems and chaos}

When the lateral size of the container is of the order of its height, hence the aspect-ratio defined as $\Gamma=\ell / h$ (where $\ell$ is some typical horizontal scale) is of order one, the structures of the convection modes strongly depend on its geometry. The flow field can be analyzed in terms of a small number of elementary motions characterizing the recirculating cells and to which individual amplitudes can be attached. This directly leads to an interpretation of the observed dynamics in terms of couplings between these variables. Formally, the success 
of this approach rests on the appropriateness of the strategy of reduction to the center manifold that expresses Haken's slaving principle [17] in mathematical terms. Basically, this "principle" says that, among the infinitely many degrees of freedom accounting for a continuous medium, most can be eliminated owing to dissipation that smoothes out all high-frequency small-scale motions to leave but but few slowly evolving fundamental modes. This fundamental property opens the way to an abstract analysis using the whole vocabulary and techniques of dynamical systems theory. In practice, the reduction is however analytically out of reach, so that the understanding gained might sound as a conjecture about how things really happen. The beauty and the strength of that conjecture rest on the applicability of the concepts brought forward by the mathematical theory, especially their universal contents.

In fact, RB convection has concentrated a large part of the efforts in the study of nontrivial features of nonlinear dynamics as applied to physical problems, namely chaos, transition scenarios, strange attractors, and the empirical reconstruction of experimental nonlinear dynamics. The main routes to chaos predicted by theory have been observed, the sub-harmonic cascade [18], the twoperiodic route and its frequency lockings [19], several types of intermittency, and even less generic situations such as quasi-periodic regimes with four or five frequencies. It turns out that, while one is unable to predict which scenario will take place in a given situation, when the system is engaged in a given wellidentified route, it strictly follows that route in its most intricate mathematical properties until unavoidable experimental limitations enter to blur the details. Here is shown the example of type III intermittency [20], the intermittency that develops beyond a sub-critical sub-harmonic bifurcation. This scenario can be modeled by means of an iteration [21]:

$$
X_{n+1}=-(1+r) X_{n}-X_{n}^{3}
$$

where $X$ represents the amplitude of the departure from a limit cycle (corresponding to the fixed point of the iteration at $X=0$ ) and $r$ is a control parameter (negative below threshold, positive above). This local map has to be completed by a global assumption about the nature of manifold on which this reduced dynamics takes place and regarding the return of escaping iterates in the vicinity of the fixed point. Type III intermittency was observed by Dubois et al. $[20]$ in convection using silicone oil (large $P$ ). A typical time series is displayed in Fig. 7 (top). Displayed on the bottom line of that figure, the return maps of the maxima $I_{n}$ of some observable plotted every two determinations, i.e. $I_{n+2}$ as a function of $I_{n}$ and the statistics of the durations of closely periodic sequences before escape - the so-called laminar intermissions - both agree quantitatively with corresponding theoretical predictions after appropriate empirical rescaling.

In the same spirit, Takens' methods of delays [22] has been used extensively to reconstruct attractors [19] and determine quantities such as fractal dimensions and Lyapunov exponents. An early example is shown in Fig. 8, again taken from 

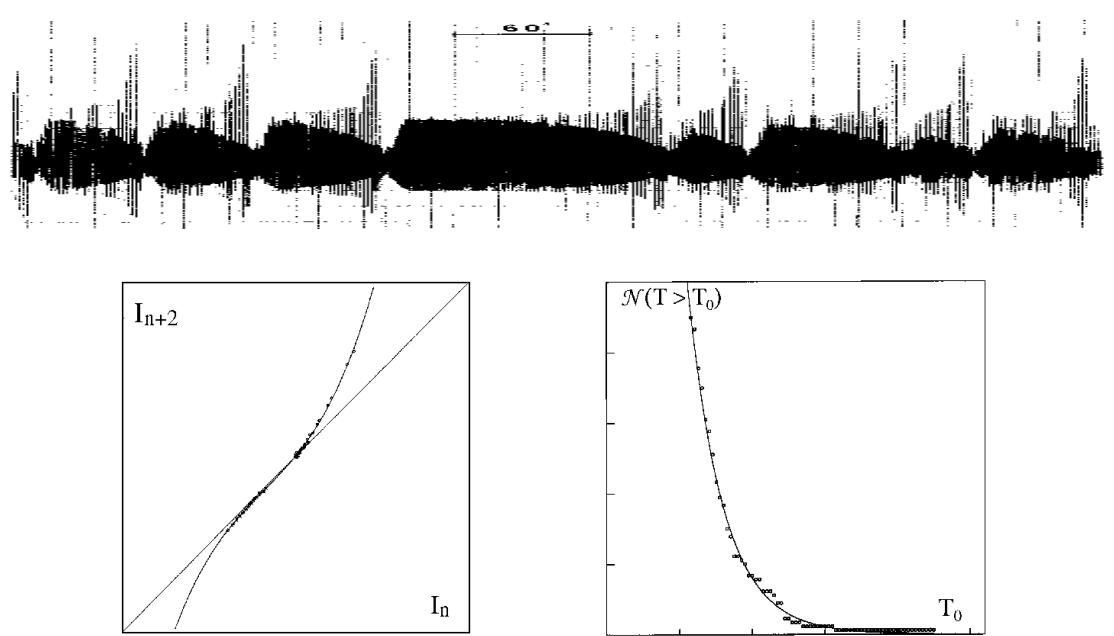

Fig. 7. Type III intermittency in RB convection after Dubois et al. [20]. Top: time series of a velocity component measured at some point in the cell. Bottom-left: effective iteration obtained by displaying maxima of that variables every two steps. Bottomright: cumulative distribution function of the duration of laminar intermissions.

the work of the Saclay group [23]. There, the correlation dimension ${ }^{3}$ [24] has been determined from the so-called correlation integral (left) computed with reconstructions at ever larger embedding dimensions $d_{\mathrm{e}}$ and the saturation observed (right) for $d_{2}=2.8$ clearly indicates the low dimensionality of the corresponding chaotic attractor.

These approaches in terms of dynamical systems with few degrees of freedom culminated with the study of the universality and the multi-fractal properties of chaos emerging from a two-periodic regime observed by Jensen et al. [25] in a convection experiment with a conducting fluid (mercury) under periodic forcing by a variable magnetic field. The left part of Fig. 9 displays a section of the two-periodic attractor at the margin of chaos and its right part the corresponding "f-of-alpha spectrum" characterizing the distribution of singularities of the distribution of points along the section.

It should again be stressed that the understanding of the transition to temporal chaos has not been obtained ab initio from the primitive problem, e.g. by truncating a Galerkin expansion as done to obtain the celebrated Lorenz model [26] (or its higher dimensional generalizations), which is analytically tractable only at the price of non-physical boundary conditions (stress-free at top

\footnotetext{
3 This quantity is often used to characterize the fractal component of strange attractors. It is equivalent to the Renyi dimension $d_{2}$. The Renyi dimensions $d_{q}$ are defined by $d_{q}=\lim _{\epsilon \rightarrow 0} \log \left(\sum_{i} p_{i}^{q}\right) / \log (\epsilon)$, where $\epsilon$ measure the size of balls covering the fractal set and $p_{i}$ the occupation probability of ball $i$ belonging to the covering of the set. From their usual definitions, it is easily shown that the fractal dimension (or capacity), the information dimension, and the correlation dimension are obtained as $d_{q}$ with $q=0,1$, and 2 , respectively.
} 

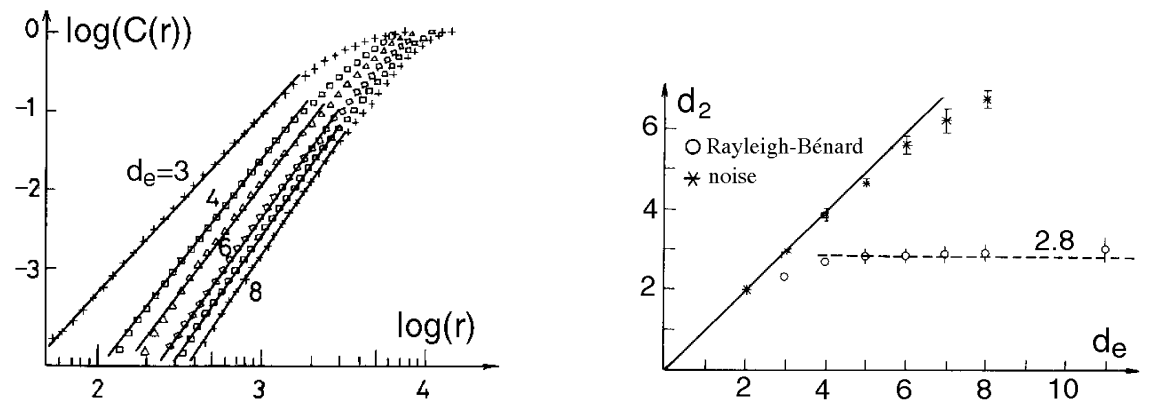

Fig. 8. Determination of fractal dimensions by Malraison et al. from Saclay RB convection data. Left: Grassberger-Procaccia correlation integral $C(r)$ as a function of the distance $r$ in embedding spaces with dimensions $d_{\mathrm{e}}$ ranging from 3 to 8 . Right: correlation dimension (slope of $C(r)$ in log-log coordinates) for the RB experiment saturating at $\nu=2.8$ (for comparison: increase observed for a synthetic noise signal). After [23].
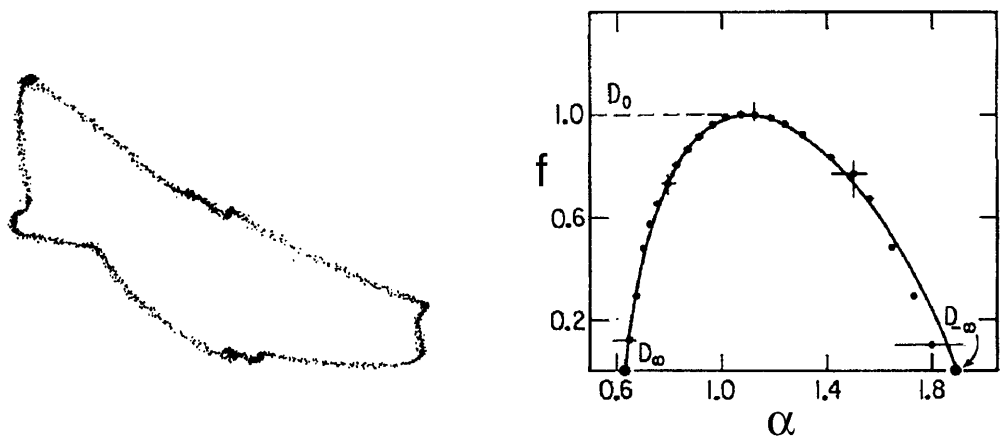

Fig. 9. Multi-fractal properties of the attractor at the threshold of the two-periodic route to chaos after Jensen et al. [25]: Left: section of the attractor. Right: f-of-alpha spectrum.

and bottom, horizontally periodic). Rather, an inductive type of modeling has been developed, resting on mathematical properties with universal contents in the dynamical systems sense, i.e. normal forms for bifurcations and their consequences. This phenomenological approach involving generic dynamical systems, especially iterations, led to impressive results on a local scale in phase space but, as a matter of fact, beyond threshold the global phase-space structure of a confined RB convection system with realistic boundary conditions becomes extremely complicated. This holds true in particular for large- $P$ fluids at high $R$, in which thin fluctuating internal thermal boundary layers were observed as illustrated in Fig. 10, while temporal-chaos concepts should remain relevant to the dynamics. 

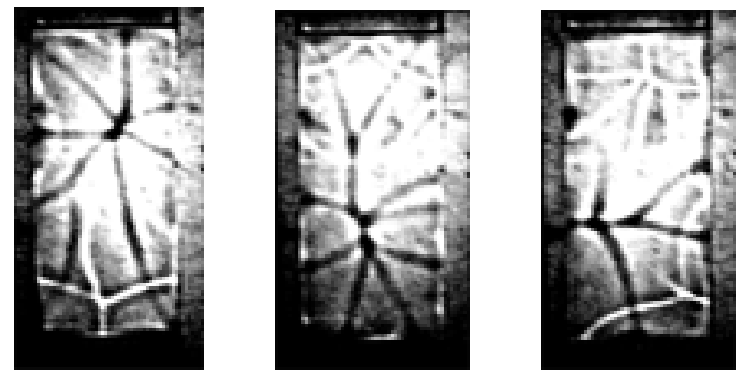

Fig. 10. Complicated patterns in silicone oil at high $R$. Adapted from [28], courtesy of F. Daviaud (Saclay).

\section{Patterns in extended systems at large $\boldsymbol{P}$}

Furthermore, coexistence of separate attraction basins for different scenarios appeared to be the rule. For example, Type I intermittency and the two-periodic route were observed in the same experimental container with the same fluid (silicone oil) but starting with initial conditions built according to different protocols [27].

In the case of confined systems, the validity of universality concepts is backed by the reduction to low-dimensional dynamical systems through adiabatic elimination of slaved variables. Appropriate adaptations are clearly needed for weakly confined systems. On general grounds, lateral confinement effects are expected to scale as $1 / \ell^{2}$. This turns out to be an advantage since, for sufficiently wide systems $(\Gamma \gg 1)$, interesting phenomena may happen in a narrow neighborhood of the threshold, thus accessible to perturbation techniques. The new meaning of universality for structures with many cells can indeed be approached in terms of modulations brought to uniformly periodic roll systems.

The standard multi-scale formalism [8] is the most natural framework for the study of weakly disordered patterns. Assuming that, close to the threshold, a modulated solution locally condensed on a pair of wavevectors $\mathbf{k}_{ \pm}= \pm k_{\mathrm{c}} \hat{\mathbf{x}}$ can be searched for in the form

$$
V(x, y, z, t)=A(x, y, t) \bar{V}(z) \exp \left(i k_{\mathrm{c}} x\right)+\text { c.c. }
$$

$A$ being a slowly varying envelope $\left(\partial_{t} \sim r, \partial_{x} \sim r^{1 / 2}, \partial_{y} \sim r^{1 / 4}, r=\right.$ $\left.\left(R-R_{\mathrm{c}}\right) / R_{\mathrm{c}} \ll 1\right)$. One is led to the Newell-Whitehead-Segel (NWS) equation $[29,30]$ :

$$
\tau_{0} \partial_{t} A=\left(r-g|A|^{2}\right) A+\xi_{0}^{2}\left[\partial_{x}+\frac{1}{2 i k_{\mathrm{c}}} \partial_{y y}\right]^{2} A
$$

where $\tau_{0}$ is the natural relaxation time of fluctuation and $\xi_{0}$, linked to the curvature of the marginal stability curve at threshold, is the natural coherence length accounting for the reluctance of the system to adopt a wavevector with a value different from $k_{\mathrm{c}}$. 


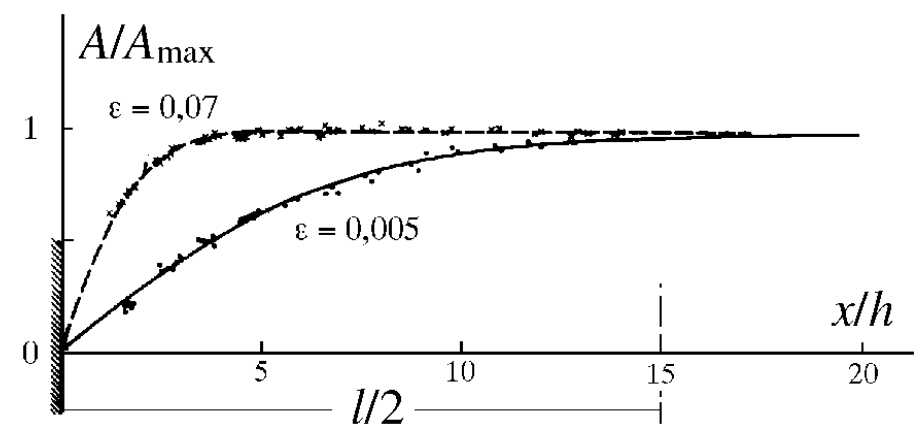

Fig. 11. The amplitude of rolls parallel to a lateral wall follows the hyperbolic-tangent law predicted by the Ginzburg-Landau theory up to the quantitative level according to the measurements reported in [31].

This equation was obtained for stress-free boundary conditions but can be shown to hold in the no-slip case with coefficients that can be computed and checked against experiments. As seen in Fig. 11, the modulation of the convection amplitude close to a lateral boundary parallel to the roll axis predicted by the theory is in excellent agreement with that experimentally measured by Wesfreid et al. [31] in a long and narrow parallelepipedic container using high- $P$ silicone oil. An equally good quantitative agreement was observed for other physical properties, e.g. the relaxation rate of fluctuations (critical slowing down), showing that the classical Landau theory of second-order phase transitions applies also to supercritical bifurcations in extended media. ${ }^{4}$

In the vicinity of the convection threshold, a large body of results about the dynamics of disordered patterns, also called textures, can be understood within the envelope formalism [33]. This remains true as long as the textures behave relaxationally, which is the case when $P \gg 1$. A single scalar envelope field can then be used, that is locally governed by the NWS equation. Owing to its real coefficients, this equation derives from a potential so that solutions to it relax towards essentially time-independent states. Solutions may be disordered, with curvature and defects, owing to the fact that they have to accommodate contradictory requirements, the most important ones being that the roll axis be perpendicular to the lateral boundaries and that the local wavelength be roughly constant and equal to its critical value in the bulk. Even in the limit $P \rightarrow \infty$, the envelope formalism is difficult to handle when textures are strongly disordered [34]. An alternative is the recourse to simplified models such as the Swift-Hohenberg (SH) model [35]:

$$
\tau_{0} \partial_{t} w=r w-\xi^{4}\left(\nabla^{2}+k_{\mathrm{c}}^{2}\right)^{2} w-g w^{3},
$$

\footnotetext{
${ }^{4}$ Corrections to the classical behavior due to fluctuation effects similar to those in thermodynamic phase transitions are not detectable in most practical situations. These effects have been recently studied by Oh and Ahlers [32].
} 


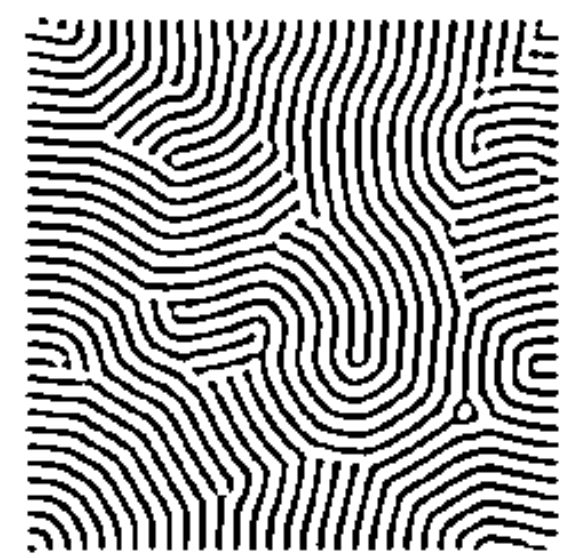

Fig. 12. Time independent textured state obtained by simulation of the SH model for $r=0.3$ in a large square domain with boundary conditions $w=\nabla_{\perp} w=0$ where $\nabla_{\perp}$ is the gradient along the normal to the boundary $\left(k_{\mathrm{c}}=1, \ell=200\right)$.

where $w$ is now a two-dimensional field function of time and the horizontal coordinates representing the local convection motion (e.g. the temperature at mid-height). The SH model was obtained by a semi-rigorous elimination of the vertical dependence through a Galerkin expansion of the thermo-hydrodynamic fields in the stress-free case. The original SH model, with its cubic nonlinearity, derives from a potential and, as such, can only lead to time-independent textures. A typical simulation result obtained by myself is displayed in Fig. 12, illustrating the frustration of geometrical origin with "grains" of well-oriented rolls and several kinds of defects joining them. Non-variational corrections can be expected however, leading to unsteady textures with very slow residual time dependence, at least as long as $P$ is sufficiently large. Accordingly, the transition to turbulence can mostly be interpreted in terms of a "fusion" of two-dimensional (scalar) textures. Variants of the SH model have been proposed to deal with convection in different circumstances, when hexagons or squares are expected owing to nonBoussinesq or heat-conductivity effects [36]. When $P$ decreases, the situation is more complicated because the velocity field recovers its rights to control the dynamics. Turbulence is then seen to occur at moderate distance of the primary convection threshold (see Fig. 6). A theoretical digression is however necessary before I come back to this problem.

\section{Weak turbulence in extended systems at small $P$}

Within the stress-free model, Siggia \& Zippelius [37] have shown that the NWS envelope equation must be corrected at lowest order to account for drift flows induced by the curvature of rolls. They obtained a set of two coupled equations for the amplitude $A$ and the intensity $U$ of the drift flow. The effect of the latter 
is to push the rolls, hence a modified NWS equation

$$
\tau_{0}\left(\partial_{t}+i k_{\mathrm{c}} U\right) A=\left(r-g|A|^{2}\right) A+\xi_{0}^{2}\left[\partial_{x}+\frac{1}{2 i k_{\mathrm{c}}} \partial_{y y}\right]^{2} A
$$

the drift flow $U$ arising from the large-scale vertical vorticity $\Omega=-\partial_{y} U$ induced by the curvature of the rolls according to

$$
\gamma \partial_{t} \Omega-\partial_{y y} \Omega=g \partial_{y}\left[A^{*}\left(\partial_{x}+\frac{1}{2 i k_{\mathrm{c}}} \partial_{y y}\right) A+\text { c.c. }\right],
$$

where $g \propto(1+P) / P^{2} \sim 1 / P$ for $P \ll 1$ and 'c.c.' denotes the complex-conjugate term. By contrast with the NWS equation, the new system does not derive from a potential so that a less trivial dynamics can develop. ${ }^{5}$ Systematic expansions in this vein have been developed by Decker and Pesch [38] for the realistic case of no-slip boundary conditions leading to an account of stability properties of the rolls in the neighborhood of the threshold in agreement with Busse's previous numerical findings [7] (and analytical results by Piquemal and myself for the zigzag instability [39]).

Close to the threshold, but not asymptotically close to it, the stability of wellaligned patterns can be studied within the phase formalism [40]. Technically the full solution, symbolically written $V(x, y, t)$, is searched for in the form $V_{0}\left[k_{0}(x+\right.$ $\phi(x, y, t))]+V_{1}+\ldots$, where $\phi$ is a slowly varying phase and $x$ the coordinate along the wavevector of the reference roll system. A compatibility condition for $\phi$ leads to a diffusion equation:

$$
\partial_{t} \phi=D_{\|} \partial_{x x} \phi+D_{\perp} \partial_{y y} \phi
$$

where the diffusion coefficients $D_{\|}$and $D_{\perp}$ are functions of the wavevector $k_{0}$ different from $k_{\mathrm{c}}$ (but not too far from it) and the relative distance to threshold $r$. Universal secondary instabilities occur when these diffusion coefficients become negative (Eckhaus when $D_{\|}<0$; zigzags when $D_{\perp}<0$ ). The generalization of this approach is due to Newell and Cross [34] who derive the diffusion equation in a form independent of a reference frame linked to the local roll wavevector. The solution being now written as $V_{0}(\Theta(x, y, t))=A(x, y, t) \exp (i \Theta(x, y, t))$, the local wavevector is given by $\mathbf{k}_{\mathrm{h}}(x, y, t)=\nabla_{\mathrm{h}} \Theta(x, y, t), k \equiv \sqrt{\mathbf{k}_{\mathrm{h}}^{2}}$, the amplitude $A$ is enslaved to the phase $\Theta$ through an eikonal equation $A=A(k)$ and $\Theta$ is governed by:

$$
\tau(k) \partial_{t} \Theta(x, y, t)+\nabla_{\mathrm{h}} \cdot\left[\mathbf{k}_{\mathrm{h}} B(k)\right]=0,
$$

where $\tau(k)$ and $B(k)$ are two functions of $k$. The previous diffusion equation is recovered when a nearly uniform roll pattern is assumed, which yields $D_{\|}=$ $-\tau^{-1} \mathrm{~d}(k B) / \mathrm{d} k$ and $D_{\perp}=-\tau^{-1} \mathrm{~d} B / \mathrm{d} k$. Cross and Newell then added drift

\footnotetext{
${ }^{5}$ The Galilean invariance of the stress-free Boussinesq problem is broken when no-slip boundary conditions are considered but, when $P$ is sufficiently small, this invariance is approximately restored beyond threshold through thin viscous boundary layers at the top and bottom plates so that the approach still makes sense.
} 
flows phenomenologically through the change: $\partial_{t} \mapsto \partial_{t}+\mathbf{U} \cdot \nabla_{\mathrm{h}}$. The field $\mathbf{U}$ that generalizes the variable $U$ introduced earlier now describes a horizontal incompressible flow deriving from a stream function $\Psi$ (i.e. $\mathbf{U} \equiv\left(\partial_{y} \Psi,-\partial_{x} \Psi\right)$ ) governed by

$$
\nabla^{2} \Psi=\gamma \hat{\mathbf{z}} \cdot \nabla_{\mathrm{h}} \times\left[\mathbf{k}_{\mathrm{h}}\left(\nabla_{\mathrm{h}} \cdot\left(\mathbf{k}_{\mathrm{h}} A^{2}\right)\right)\right]
$$

where $A$ is the amplitude and $\hat{\mathbf{z}}=\hat{\mathbf{x}} \times \hat{\mathbf{y}}$. In practice, in the unstable range, generalized phase equations form an ill-posed problem leading to singularities and some regularization is demanded, as discussed in detail by Newell and coworkers (see, e.g. [8]).

These theoretical problems are indeed fully relevant to the understanding of the transition to turbulence in low Prandtl number fluids that do not behave relaxationally but have more active dynamics of inertial origin. Whereas it was known for long from conventional studies that turbulent states can be observed at moderate $R$ when $P \sim 1$ or below, see Fig. 6 , it was later discovered that the range of strictly time-independent convection was extremely narrow at large aspect-ratios $\Gamma \gg 1$ [41]. However, the kind of time-dependence that developed was not real high-frequency turbulence but resembled a low-frequency broadband noise with a power-law spectrum appearing before any trace of secondary instability [42]. This behavior was not well understood until Pocheau et al. [43] showed that this noise resulted from a cycle involving the nucleation, migration, and annihilation of dislocations. Pictures of this cycle are displayed in Fig. 13.

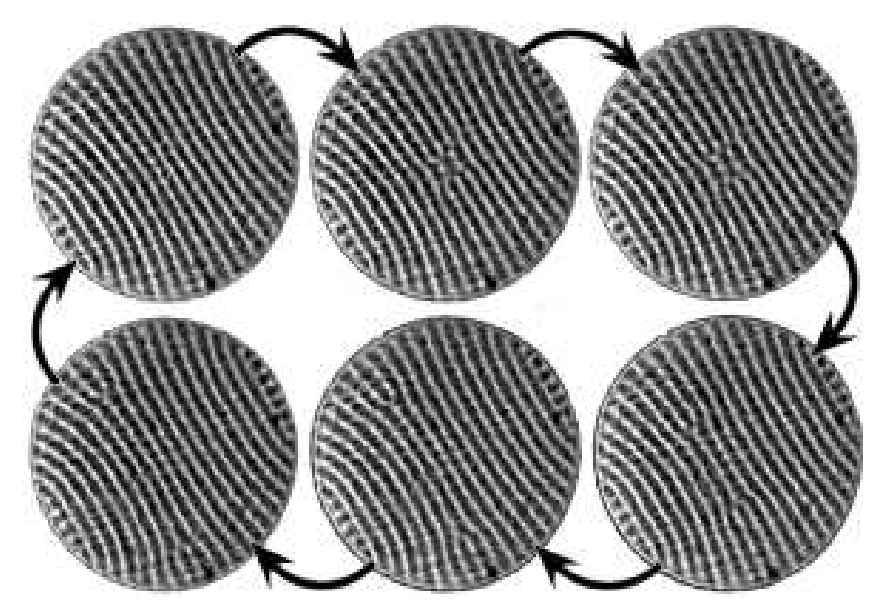

Fig. 13. In a cylindrical container (with Argon under pressure as the working fluid), lateral boundary effects on the roll orientation at the walls imply some curvature in the buck. Interplaying with drift flow, a periodic process of nucleation, migration, and annihilation of dislocations develops, at first roughly periodic in time. Irregularities in the process lead to a noisy dynamics with power-law spectrum. Pictures kindly provided by V. Croquette (ENS-Ulm), see [44] for details. 
In an attempt to get a semi-microscopic account of convection at low Prandtl number in weakly confined systems, it seems necessary to include drift flow effects in the SH model. Paralleling Siggia and Zippelius, I derived a generalized SH model by truncating at lowest significant order a Galerkin expansion of the thermo-hydrodynamic fields while taking care of the large scale flow driven by curvature effects, obtaining [45, a]:

$$
\begin{aligned}
\partial_{t} w+\mathbf{U} \cdot \nabla_{\mathrm{h}} w & =\left[r-\left(\nabla_{\mathrm{h}}^{2}+1\right)^{2}\right] w-\mathcal{N}(w), \\
\left(\partial_{t}+P \nabla_{\mathrm{h}}^{2}\right) \nabla_{\mathrm{h}}^{2} \Psi & =\partial_{y} w \partial_{x} \nabla_{\mathrm{h}}^{2} w-\partial_{x} w \partial_{y} \boldsymbol{\nabla}_{\mathrm{h}}^{2} w
\end{aligned}
$$

where, as above, $\Psi$ is the stream function from which the velocity field $\mathbf{U}$ derives and $\mathcal{N}(w)$ some nonlinear saturating term (either $w^{3}$ as in the original $\mathrm{SH}$ model, or e.g. $\left[\left(\boldsymbol{\nabla}_{\mathrm{h}} w\right)+w^{2}\right] w$ as in $[45, \mathrm{a}]$, or possibly more general - even nonlocal- expressions). Numerical simulations of the extended SH model [45, b] have led to an interpretation of weak turbulence in extended RB convection systems in terms of a dynamical compatibility of drift flows to the spatially disordered topology of patterns implied by the geometrical frustration imposed by the lateral boundaries.

At larger but still small $P$, a quite different kind of weak turbulence, called spiral defect chaos (SDC) has also been observed by Ahlers and coworkers [46] among others. This regime is illustrated in Fig. 14 for two different values of $R$. Bistability with respect to this regime has also to be noted: at the same $R$, straight rolls are still locally stable but, when it is nucleated somewhere, the SDC regime invades the whole surface of the system at the expense of the rolls. From a modeling viewpoint, the SDC regime has been observed in simplified systems such as the generalized SH model presented above [47] or others simplified models in the same spirit [48]. The role of drift flows seem essential for such a spacetime chaotic behavior but, on more general ground, its extensive character (i.e. a density of spiral cores can be defined, independent of the shape of the container provided it is sufficiently wide) makes it interesting from the point of view of a statistical analysis of out-of-equilibrium systems at the thermodynamic limit.
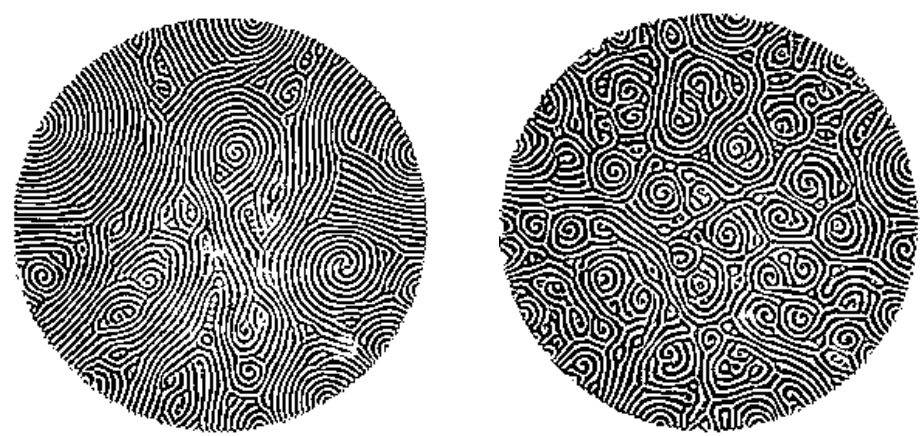

Fig. 14. The density of spiral cores increases as $R$ gets farther beyond the threshold of SDC. Pictures kindly provided by G. Ahlers (UCSB), see [46]. 


\section{Space-time intermittency and statistical physics}

Another situation is of interest with respect to statistical aspects of the transition to turbulence when confinement effects are exploited to produce a quasi-onedimensional pattern, see Fig. 15. In the case to be described, the fluid is silicone oil with moderate to high Prandtl number so that no strong large-scale flow effects are expected. Seen from above the positions of the thin thermal boundary layers between consecutive cells can be recorded as a function of time to construct space-time diagrams. The most interesting experiments have been performed in the narrow annular cell shown in Fig. 16 (top), thus avoiding parasitic end effects.

The transition to turbulence in this system happens via a new specific scenario: the time-independent regular arrangement of cells below the transition is disrupted by intermittent chaotic bursts above threshold. At a given time the system can be divided in laminar and turbulent domains and at a given point in space the system is alternatively laminar or turbulent, hence the term spacetime intermittency (STI). This kind of transition has been observed in several convection experiments $[49,50]$ but also in other physical systems. For a review, consult [51].

The theoretical account of this scenario follows Pomeau's idea [53] of an equivalence of STI with a time-oriented stochastic process known as directed percolation in statistical physics. This process deals with the modeling of epidemic processes in which subjects in good health (in the so-called absorbing state) are contaminated by some disease (the excited state) with finite local probability. The subjects stand at the nodes of a lattice and contamination is from one node to its neighbors while time is advancing by steps. Above some probability threshold, contamination is sustained and propagates to infinity with finite probability, otherwise the epidemics ceases spontaneously. Directed percolation is a critical phenomenon that defines a so-called universality class, with a specific set of critical exponents, controlling, e.g. the fraction of excited states

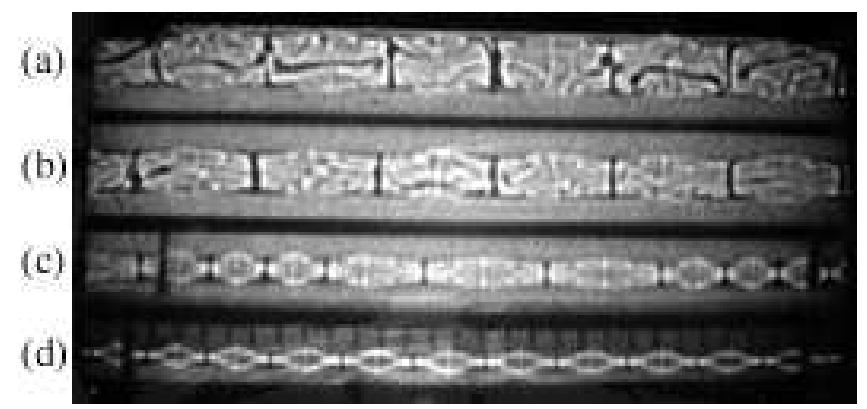

Fig. 15. Convection in four parallel slits with decreasing width from (a) to (d), seen from above. The linear arrangement of cells with complex individual flow patterns observed in (a) is similar to those in Fig. 10. In (d) a regular row of simpler cells has set in, hence a quasi-one-dimensional pattern with fewer local degree of freedom. Adapted from [28], courtesy of F. Daviaud (Saclay). 

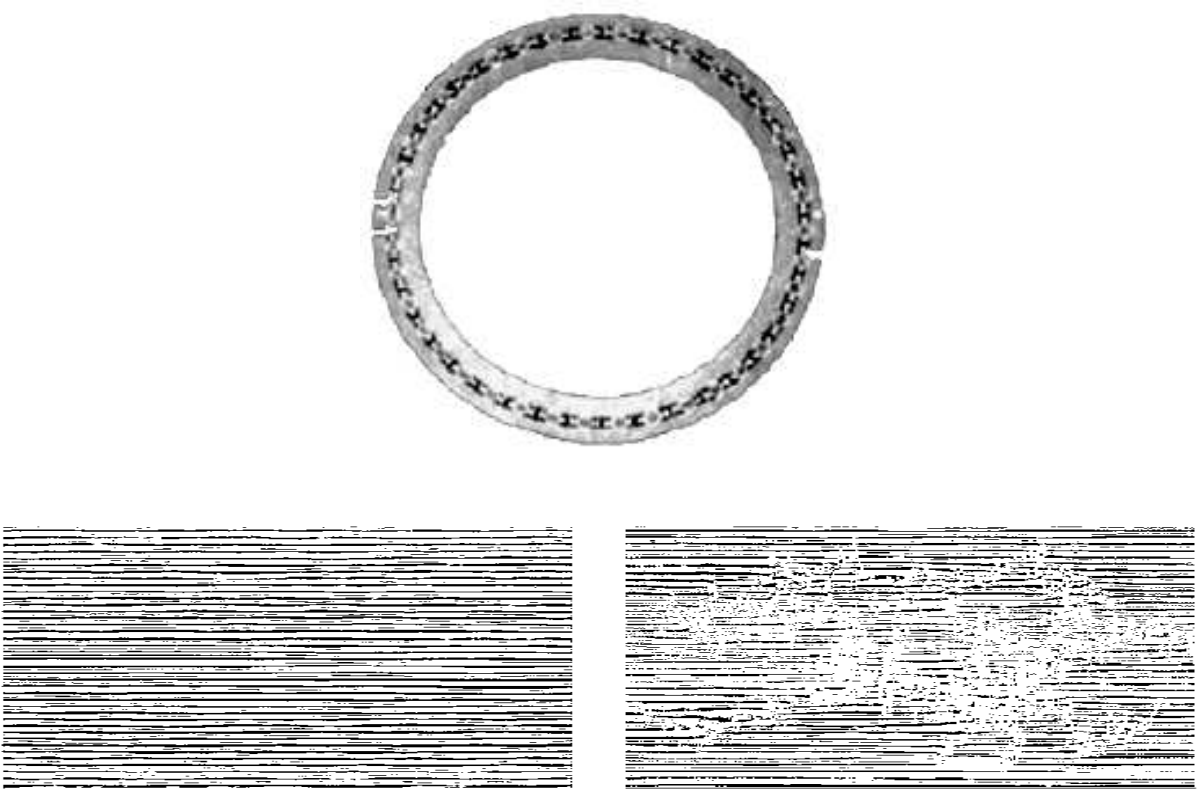

Fig. 16. Transition to space-time intermittency in quasi-one-dimensional geometry. Top: annular convection cell, seen from above [the radial aspect ratio is similar to the transverse one in Fig. 15 (d)]. Bottom: space-time diagrams with the azimuthal coordinate along the vertical and time running from left to right, below (left) and above (right) the STI threshold. Courtesy of F. Daviaud (Saclay), see [28] for details.

that grows as a power law of the distance to threshold.

An essential assumption is that, out of the two possible coexisting states at each location, the one playing the role of the absorbing state is locally stable so that there cannot be spontaneous birth of excited states. Coexistence of states in the local phase space usually manifests itself by the formation of walls for systems distributed in space. In the simplest case of a potential system these walls move so as to minimize the potential. In the more complicated case where the excited state is a chaotic transient, the motion of the wall through contamination becomes random. In order to better understand how STI can occur in deterministic systems, that is to say how local transient temporal chaos can be converted in sustained space-time chaos by the interaction between neighboring subsystems, models in terms of coupled map lattices [54] have been built with an appropriate local phase space structure [55]. The universality issue turns out to be intricate, especially regarding the thermodynamic limit of infinitely large systems in the long-time limit, after transients have decayed [56]. As far as convection in quasi-one-dimensional is concerned, turbulence is obviously the excited state. The statistics of the size of turbulent domains has been studied as a function of $R$, showing that the STI transition was only imperfect [50] due to a tiny probability of spontaneous nucleation of turbulent cells (possibly linked 
to the focusing of a long-wavelength secondary instability of the convection cell chain).

\section{Convection at large Rayleigh numbers}

Beyond the transitional stage, convection enters a fully developed turbulent regime usually best characterized by physical properties scaling as some power of $R$, as noticed by Siggia [11]. Most often one is interested in the behavior of the Nusselt number defined as

$$
N=\frac{\text { total heat flux }}{\text { conduction heat flux }}
$$

where the "total heat flux" is the measured flux and the "conduction heat flux" is the flux computed from the applied temperature difference upon assuming Fourier law in a fluid at rest, hence $N=1$ below the primary threshold $\left(R<R_{\mathrm{c}}\right)$, $N-1$ measuring the contribution of convection beyond. In the weakly nonlinear regime close to threshold it is expected to vary as

$$
N-1 \propto v_{z} \theta \propto \frac{R-R_{\mathrm{c}}}{R_{\mathrm{c}}},
$$

since $N-1$ is the average over the cell of the product $\theta v_{z}$, and each term scales as $\left[\left(R-R_{\mathrm{c}}\right) / R_{\mathrm{c}}\right]^{1 / 2}$. This behavior has been well observed experimentally long ago and, as mentioned by Chandrasekhar [5], can serve to locate the threshold with precision. When $R$ becomes larger than few hundreds of $R_{\mathrm{c}}, N$ increases with $R$ as a power law:

$$
N \sim R^{\gamma}
$$

but some confusion exists about the relevant value of $\gamma$ that may depend on the range of $R$ considered, with crossovers between different regimes, the nature of the fluid (value of $P$ ) and to a lesser extent on the shape of the container or the roughness of the top and bottom plates. Exact bounds can be given for $\gamma$ (see [57] for references) and early arguments predicted $\gamma=1 / 3$ or $\gamma=1 / 2$. The first value is obtained by assuming that the turbulent heat flux is fully controlled by finite-width thermal boundary layers so that it becomes independent of the container height (Malkus, 1958) while the second one, considered as the "ultimate regime" at asymptotically large $R$, is reached when buoyancy is fully balanced by advection in the momentum equation so that the heat transfer no longer depends on molecular properties $\kappa$ and $\nu$ (Kraichnan, 1962). Consult [58] for an introductory presentation and [11] or [59] for more information. Some early experiments seemed to support $\gamma=1 / 3$ while others for $P \ll 1$ yielded rather $\gamma=1 / 4$, As a matter of fact, these early studies were performed in extended geometry with many convection cells and did not allow to test sufficiently large ranges of parameters. Since 1985 new experiments specifically focusing on this problem have been developed, see for example [60-62] among many others. In order to reach high values of $R$ without increasing the temperature difference 


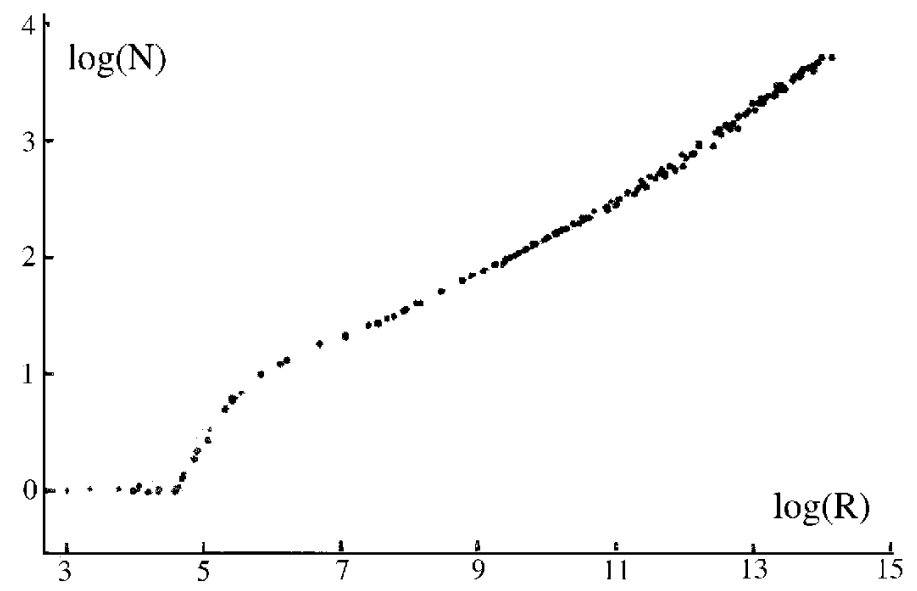

Fig. 17. Total heat flux as measured in terms of the Nusselt number as a function of the Rayleigh number (in log-log scale). After the initial abrupt increase corresponding to the weakly nonlinear regime, the Nusselt number varies more gently as $R^{\gamma}$. The exponent is close to $2 / 7$ over a four-decade range between $10^{7}$ and $10^{11}$, and somewhat larger, about $1 / 2$, above. Adapted from Chavanne et al. [61, a].

too much (validity condition for the Boussinesq approximation), containers have indeed to be tall since, from its definition $R$ grows as $h^{3}$ at given $\Delta T$. But large aspect ratios cannot be maintained if the experiment is to stay within reasonable horizontal-size limits. In practice aspect ratios $\Gamma=1 / 2$ or 1 have been used and very large $R$, up to $10^{18}$ times the critical value, have been achieved. The drawback of small aspect ratios is that the mean large scale flow may control an important part of the heat transfer, especially by producing a "wind" along the top and bottom plates. For example thermal transfer through so created velocity boundary layer yields $N \sim R^{1 / 4}$ when they are laminar and $N \sim R^{2 / 7}$ when they are turbulent.

Figure 17 adapted from the results of Chavanne et al. in liquid helium [61], is typical of the most recent experiments. One can easily identify the previously mentioned weakly nonlinear regime close to threshold, ${ }^{6}$ then the "soft turbulence" regime where chaos is still mostly temporal, next "hard turbulence" with an exponent $\gamma \simeq 2 / 7$ explained by the theory involving thermal transfer through turbulent layers sheared by the general circulation wind, and finally the "ultimate" regime with exponent tending to $1 / 2$. The existence of this last regime has been challenged [60] and conditions for its observation clarified by Roche et al. $[61, \mathrm{c}]$.

The different turbulence regimes have been reconsidered by Grossmann and Lohse [59] who distinguish them from the origin of the main contributions to the dissipation, in the bulk or within boundary layers and give a unified scaling picture of the strong turbulence problem in RB convection. According to this

${ }^{6}$ The threshold is shifted somewhat beyond 1708 owing to small aspect-ratio effects. 
picture, four main regimes can exist with different $\gamma$ signatures and it is argued that the scaling with $\gamma=2 / 7$ though observed over a rather wide range of Rayleigh numbers (and in spite of its appealing physical interpretation in terms of heat transfer through turbulent boundary layers) is equally well accounted for as a blend of regimes with $\gamma=1 / 4$ and $\gamma=1 / 3$ with appropriate weighting coefficients. How these regimes depend on the Prandtl number (also possibly on the shape of the container and associated bifurcations of the mean large scale flow) is the subject of recent studies, e.g. [62].

\section{Conclusion}

One century after his first experiments, one cannot but notice that Bénard opened a particularly rich field of research, accessible to detailed analysis both theoretical and experimental. Once the initial misunderstanding about the role of surface tension has been cleared up, RB convection (within the Boussinesq approximation) has indeed presented itself as an ideal testing ground for the interplay of mathematics and physics methods, especially during the last thirty years. In particular, fundamental problems related to universality could be tackled with, both for confined systems where the theory of dynamical systems is relevant (chaos and transition scenarios, e.g. the sub-harmonic cascade) and for extended systems where statistical physics is an appealing framework (GinzburgLandau formalism and nonlinear pattern selection, space-time intermittency and directed percolation). It should further be noticed that progress has been obtained through an exemplary feedback process involving experiments, theory, and modeling. Let us hope that the methods developed to reach such an improved understanding of the emergence of complexity in this specific physical system will also fuel the study of issues crucial to the future of our natural environment.

\section{References}

1. H. Bénard, "Les tourbillons cellulaires dans une nappe liquide," Rev. Gén. Sci. pures et appl. 11 (1900) 1261-1271 \& 1309-1328.

2. Lord Rayleigh, "On the convective currents in a horizontal layer of fluid when the higher temperature is on the under side," Phil. Mag. 32 (1916) 529-546.

3. J.R.A. Pearson, "On convection cells induced by surface tension," J. Fluid Mech. 4 (1958) 489-500.

4. P. Cerisier, C. Jamond, J. Pantaloni, J.C. Charmet, "Déformation de la surface libre en convection de Bénard-Marangoni," J. Physique 45 (1984) 405-411.

5. S. Chandrasekhar, Hydrodynamic and hydromagnetic stability (Clarendon Press, Oxford, 1961)

6. E.L. Koschmieder, Bénard cells and Taylor vortices (Cambridge University Press, 1993).

7. F.H. Busse, "Non-linear properties of thermal convection," Rep. Prog. Phys. 41 (1978) 1929-1967. and "Transition to turbulence in thermal convection," in Convective transport and instability phenomena, J. Zierep, H. Oertel, jr., eds (Braun, Karlsruhe, 1982). 
8. A.C. Newell, Th. Passot, J. Lega, "Order parameter equations for patterns," Ann. Rev. Fluid Mech. 25 (1993) 399-453.

9. M.C. Cross, P.C. Hohenberg, "Pattern formation outside of equilibrium," Rev. Mod. Phys. 65 (1993) 851-1112.

10. E. Bodenschatz, W. Pesch, G. Ahlers, "Recent developments in Rayleigh-Bénard convection," Ann. Rev. Fluid Mech. 32 (2000) 708-778.

11. E.D. Siggia, "High Rayleigh number convection," Annu. Rev. Fluid Mech. 26 (1994) 137-168.

12. W.V.R. Malkus, G. Veronis, "Finite amplitude cellular convection," J. Fluid Mech. 38 (1958) 227-260.

13. A. Schlüter, D. Lortz, F.H. Busse, "On the stability of finite amplitude convection," J. Fluid Mech. 23 (1965) 129-144.

14. R. Krishnamurti, "Some further studies on the transition to turbulent convection," J. Fluid Mech. 60 (1973) 285-303.

15. D. Ruelle, F. Takens, "On the nature of turbulence," Commun. Math. Phys. 20 (1971) 167-192. Addendum 23 (1971) 344.

16. L.D. Landau, "On the problem of turbulence," reprinted in Collected Papers of L.D. Landau, D. ter Haar, ed. (Pergamon, Oxford, 1965) pp. 387-391.

17. H. Haken, Synergetics (Springer, 1983).

18. A. Libchaber, J. Maurer, "Une expérience de Rayleigh-Bénard en gémométire réduite; multiplication, accrochage et démultiplication de fréquences," J. Physique Colloques 41-C3 (1980) 51-56.

19. M. Sano, Y. Sawada, "Experimental study on Poincaré mappings in RayleighBénard convection" in Turbulence and Chaotic Phenomena in Fluids, T. Tatsumi, ed., (Elsevier, 1984).

20. M. Dubois, M.A. Rubio, P. Bergé, "Experimental evidence of intermittencies associated with a subharmonic bifurcation," Phys. Rev. Lett. 51 (1983) 1446-1449.

21. Y. Pomeau, P. Manneville, "Intermittent transition to turbulence in dissipative dynamical systems," Commun. Math. Phys. 74 (1980) 189-197.

22. F. Takens, "Detecting strange attractors in turbulence," in Dynamical systems and turbulence, D.A. Rand, L.S. Young, eds., Lect. Notes in Mathematics 898 (Springer, 1981) pp.366-381.

23. B. Malraison, P. Atten, P. Bergé, M. Dubois, "Dimension d'attracteurs étranges: une détermination expérimentale en régime chaotique de deux systèmes convectifs," C.R. Acad. Sc. Paris 297 Série II (1983) 209-214.

24. P. Grassberger, I. Procaccia, "Measuring the strangeness of strange attractors," Physica D 9 (1983) 189-208.

25. M.H. Jensen, L.P. Kadanoff, A. Libchaber, I. Procaccia, J. Stavans, "Global universality at the onset of chaos: results of a forced Rayleigh-Bénard experiment," Phys. Rev. Lett. 55 (1985) 2798-2801.

26. E.N. Lorenz, "Deterministic nonperiodic flow," J. Atmos. Sci. 20 (1963) 130-141.

27. M. Dubois, P. Bergé, "Étude expérimentale des transitions vers le chaos en convection de Rayleigh-Bénard," in Le Chaos, Théorie et expériences, P. Bergé, ed. (Eyrolles, 1988) pp. 1-72 (in French).

28. P. Bergé, "From temporal chaos towards spatial effects," Nuclear Physics B (Proc. Suppl.) 2 (1987) 247-258.

29. L.A. Segel, "Distant side-walls cause slow amplitude modulation of cellular convection," J. Fluid Mech. 38 (1969) 203-224.

30. A.C. Newell, J.A. Whitehead, "Finite bandwidth, finite amplitude convection," J. Fluid Mech. 38 (1969) 279-303. 
31. J.E. Wesfreid, Y. Pomeau, M. Dubois, Ch. Normand, P. Bergé, "Critical effects in Rayleigh-Bénard convection," J. Physique 39 (1978) 725-731.

32. J. Oh \& G. Ahlers, "Fluctations in a fluid with a thermal gradient," UCSB preprint, September 2002.

33. M.C. Cross, "Ingredients of a theory of convective textures close to onset," Phys. Rev. A 25 (1982) 1065-1076.

34. M.C. Cross, A.C. Newell, "Convection patterns in large aspect ratio systems," Physica D 10 (1984) 299-328.

35. J. Swift, P.C. Hohenberg, "Hydrodynamic fluctuations at the convective instability," Phys. Rev. A 15 (1977) 319-328.

36. C. Perez-Garcia, J. Millán-Rodriguez, H. Herrero, "A generalized Swif-Hohenberg model for several convective problems," in Instabilities and Nonequilibrium Structures IV, E. Tirapegui, W. Zeller, eds. (Kluwer, 1993) pp. 225-234.

37. E.D. Siggia, A. Zippelius, "Pattern selection in Rayleigh-Bénard convection near threshold," Phys. Rev. Lett. 47 (1981) 835-838.

38. W. Decker, W. Pesch, "Order parameter and amplitude equations for the Rayleigh-Bénard convection,” J. Phys. II France 4 (1994) 419-438.

39. P. Manneville, J.M. Piquemal, "Zigzag instability and axisymmetric rolls in Rayleigh-Bénard convection, the effects of curvature," Phys. Rev. A 28 (1983) 1774-1790.

40. Y. Pomeau, P. Manneville, "Stability and fluctuations of a spatially periodic convective flow," J. Physique Lettres 40 (1979) L-610.

41. G. Ahlers, R.P. Behringer, "Evolution of turbulence from the Rayleigh-Bénard instability," Phys. Rev. Lett. 40 (1978) 712-716.

42. A. Libchaber, J. Maurer, "Local probe in a Rayleigh-Bénard experiment in liquid Helium," J. Physique-Lettres 39 (1978) L369-L372.

43. A. Pocheau, V. Croquette, P. Le Gal, "Turbulence in a cylindrical container of Argon near threshold of convection," Phys. Rev. Lett. 55 (1985) 1094-1097.

44. V. Croquette, "Convective Pattern Dynamics at Low Prandtl Number. Part I \& II." Contemporary Physics 30 (1989) 113-133 \& 153-171.

45. P. Manneville, (a) "A two-dimensional model for three-dimensional convective patterns in wide containers," J. Physique 44 (1983) 759-765. (b) "Towards an understanding of weak turbulence close to the convection threshold in large aspect ratio systems," J.Physique Lettres 44 (1983) L903-L916.

46. S.W. Morris, E. Bodenschatz, D.S. Cannell, G. Ahlers, "The spatio-temporal structure of spiral-defect chaos," Physica D 97 (1996) 164-179.

47. (a) H.-w. Xi, J.D. Gunton, J. Viñals, "Spiral defect chaos in a model of RayleighBénard convection," Phys. Rev. Lett. 71 (1993) 2030-2033. (b) H.-w. Xi, J.D. Gunton, J. Viñals, Study of spiral pattern formation in Rayleigh-Bénard convection Phys. Rev. E 47 (1993) R2987-R2990. (c) M. Bestehorn, M. Neufeld, R. Friedrich, H. Haken, "Comment on 'Spiral-pattern formation in Rayeigh-Bénard convection'," Phys. Rev. E 50 (1994) 625-626. (d) X.-j. Li, H.-w. Xi, J.D. Gunton, "Nature of the roll to spiral-defect-chaos transition," Phys. Rev. E 57 (1998) 1705.

48. W. Decker, W. Pesch, A. Weber, "Spiral defect chaos in Rayleigh-Bénard convection," Phys. Rev. Lett. 73 (1994) 648-651.

49. S. Ciliberto, P. Bigazzi, "Spatiotemporal intermittency in Rayleigh-Bénard convection," Phys. Rev. Lett. 60 (1988) 286-289.

50. F. Daviaud, M. Dubois, P. Bergé, "Spatio-temporal intermittency in quasi onedimensional Rayleigh-Bénard convection," Europhys. Lett. 9 (1989) 441-446.

51. F. Daviaud, "Experiments in 1D turbulence," in [52] 
52. P. Tabeling, O. Cardoso, eds., Turbulence. A Tentative Dictionary (Plenum Press, 1994).

53. Y. Pomeau, "Front motion, metastability and subcritical bifurcations in hydrodynamics," Physica D 23 (1986) 3-11.

54. For general information consult: K. Kaneko, Theory and Application of Coupled Map Lattices (Wiley, 1994).

55. (a) H. Chaté, P. Manneville, "Spatiotemporal intermittency in coupled map lattices," Physica D 32 (1988) 409-422. For a review, see (b) H. Chaté, P. Manneville, "Spatiotemporal intermittency," in [52].

56. (a) P. Grassberger, Th. Schreiber, "Phase transitions in coupled map lattices," Physica D 50 (1991) 177-188. (b) G. Rousseau, B. Giorgini, R. Livi, H. Chaté, "Dynamical phases in a cellular automaton model for epidemic propagation," Physica D 103 (1997) 554-563.

57. (a) L. Howard, "Bounds on flow quantities," Annu. Rev. Fluid Mech. 4 (1972) 473-494. (b) F.H. Busse, "The optimum theory of turbulence," Adv. Appl. Mech. 18 (1978) 77-121. (c) C.R. Doering, P. Constantin, "Variational bounds on energy dissipation in incompressible flows. III. Convection," Phys. Rev. E 53 (1996) 59575981 .

58. J. Sommeria, "The elusive "ultimate state' of thermal convection," Nature 398 (1999) 294-295 (news and views).

59. S. Grossmann \& D. Lohse, (a) "Scaling in thermal convection: a unifying theory," J. Fluid Mech. 407 (2000) 27-56. (b) "Thermal convection for large Prandtl numbers," Phys. Rev. Lett. 86 (2001) 3316-3319;

60. J.J. Niemela, L. Skrbek, K.R. Sreenivasan, R.J. Donnelly, "Turbulent convection at very high Rayleigh numbers," Nature 404 (2000) 837-840, err. 406 (2000) 439.

61. (a) X. Chavanne, F. Chillà, B. Castaing, B. Hébral, B. Chabaud, \& J. Chaussy, "Observation of the ultimate regime in Rayleigh-Bénard convection," Phys. Rev. Lett. 79 (1997) 3648-3651. (b) X. Chavanne, F. Chillà, B. Chabaud, B. Castaing \& B. Hébral, "Turbulent Rayleigh-Bénard convection in gaseous and liquid He," Phys. Fluids 13 (2001) 1300-1320. (c) P.-E. Roche, B. Castaing, B. Chabaud, B. Hébral, "Observation of the 1/2 power law in Rayleigh-Bénard convection," Phys. Rev. E 63 045303-1-4 (Rapid Communications).

62. G. Ahlers \& Xiaochao Xu, "Prandtl-number dependence of heat transport in turbulent Rayleigh-Bénard convection," Phys. Rev. Lett. 86 (2001) 3320-3323. 